\title{
Macromia legrandi n. sp., Odonate nouveau du Togo (Corduliidae)
}

\author{
A. Gauthier ${ }^{1}$
}

Mots clés : Odonata, Anisoptera, Corduliidae, nouvelle espece, Togo.

Macromia legrandi n. sp., récoltée au Togo est décrite et illustrée. Une clé de détermination est donnée pour les mâles de Macromia du groupe " funicularia ».

Macromia legrandi n. sp., a new dragonfly from Togo (Odonata, Corduliidae).

Keywords: Odonata, Anisoptera, Corduliidae, new species, Togo.

Macromia legrandi n. sp., found in Togo is described and illustrated. An identification key is provided for the males of Macromia of the "funicularia "group.

Macromia legrandi $n$. sp. est décrite et illustrée d'après deux mâles récoltés au Togo. Ce nouveau taxon appartient au groupe "funicularia" qui comprend maintenant quatre espèces : $M$. funicularia Martin, 1906, M. funicularioides Legrand, 1983, M. gamblesi Lindley, 1980 , et $M$. legrandi spec. nov. Rappelons que $M$. bredoi Schouteden, 1934, a été mise en synonymie avec $M$. funicularia par Fraser (1954). Les mâles de ces espèces pourront être différenciés à l'aide de la clé de détermination donnée à la fin du présent travail.

\section{Macromia legrandi $n$. sp.}

Matériel : 1 mâle holotype, Cascades de Kpimé, Togo, IV-1986, J. M. Bousquet leg., in coll. A Gau. thier ; 1 mâle paratype, id., in coll. MNHN., Paris.

\section{Description du mâle holotype}

- Longueur de l'abdomen (sans les appendices) : $44 \mathrm{~mm}$. Longueur de l'aile postérieure : $36.5 \mathrm{~mm}$.

\footnotetext{
1. Laboratoire d'Entomologie, Université Paul Sabatier, 118, route de Narbonne, 31062 Toulouse Cedex, France.
}

- Tête : labium jaune avec une ligne noire longitudinale au centre du lobe médian se prolongeant le long de la marge interne des palpes; une bande brunâtre oblique traverse chaque palpe d'avant en arrière et de l'interieur vers l'extérieur; face brunâtre, les côtés du postclypeus jaunes.; front et vertex brun-noir avec de forts reflets métalliques bleus : occiput noir.

- Synthorax brun, avec des reflets métalliques verts ; une strie antéhumérale et deux stries latérales jaunes, l'une placée sur le métépisternite, l'autre sur le bord postérieur du métépimérite latéral. Sinus antéalaire jaune. Pattes noires.

- Ailes hyalines, nervation, manbranule et pterostigma noirs; Anténodales et postnodales: 7 : $1415: 8$ aux ailes antérieures et $8: 11-10: 9$ aux ailes postérieures; 5 cuqs aux ailes antérieures, 4 aux postérieures : 3 veines dans les hypertriangles (2 à l'aile postérieure droite); deux rangées de cellules dans le champs discoidal ; boucle anale de six cellules; deux dans le triangle anal.

- Abdomen noir ornementé de jaune comme suit : premier segment avec une tâche de chaque côté; deuxième segment portant un collier sinueux recouvrant les oreillettes, se rétrécissant vers la base 

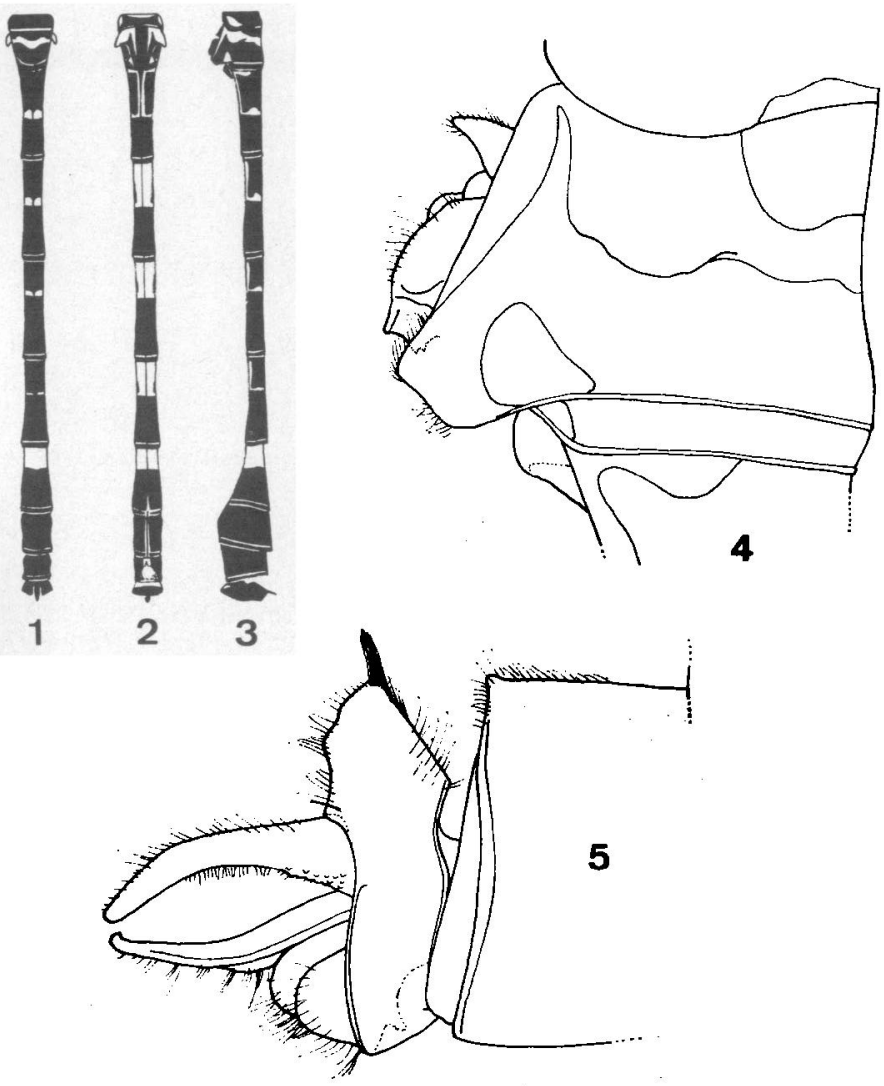

Fig. 1.5. Macromia legrandi n. sp. 1 : Abdomen, vue dorsale. 2 : idem, vue ventrale. -3 : idern, vue latérale gauche. $4:$ Second segment abdominal et genitalia, vue latérale gauche. 5 : Extrémité abdominale, vue latérale droite. 
inférieure et se projetant ensuite en arrière le long de la fosse génitale, une tâche triangulaire à la base du lobe génital (fig. 4) ; troisième segment avec de chaque côté une petite lunule latérobasale, une ligne longeant le bord inférieur du tergite jusqu'à la suture jugale où se trouve une deuxième lunule: seule la paire de lunules jugales reste sur les segments quatre à six, le dessous de ces segments est entièrement jaune de la base à la suture jugale ; septième segment jaune sur le tiers proximal ; sternite du neuvième segment jaune clair ainsi que le dessous du dixième. Appendices noirs, de même longueur que la lame supraanale, couverts de petites dents à leur base sur le côté externe (fig. 5).

- Genitalia : lobe subrectangulaire, hamulus terminé par deux dents assez petites.

\section{Paratype mâle}

Longueur de l'abdomen (sans les appendices) : $\mathbf{4 5}$ $\mathrm{mm}$; longueur de l'aile postérieure : $38 \mathrm{~mm}$. Anténodales et postnodales : $8:-16-15: 8$ aux ailes antérieures et $10: 12-11: 11$ aux ailes postérieures ; 7 cuqs à l'aile antérieure gauche, 6 à l'aile antérieure droite, 4 aux postérieures.
Zlé de détermination des mâles du groupe "funicularia".

1. Strie antéhumérale présente sur le synthorax 2

- Strie antéhumérale absente .... funicularia

2 - Appendices anaux plus longs que la lame supraanale ..................... 3

Appendices anaux pas plus longs que la lame supraanale ............... legrandi

3 - Harnulus des genitalia terminé par une seule dent $\ldots \ldots \ldots \ldots \ldots \ldots$ funicularioides

- Hamulus des genitalia terminé par deux petites dents .................. gamblesi

C'est un grand plaisir pour moi de dédier cet te nouvelle espèce a mon collègue Jean Legrand du Muséum National d'Histoire Naturelle de Paris.

\section{Travaux cités}

Fraser (F.C.). 1954, - New species of Macromia from tropical Africa. Revue Zool. Bot. afr, $49: 41-76$.

Legrand (J.). 1983. - Note sur les Odonates actuellement connus des Monts Nimba (Afrique occidentale). Revue fr. Ent. (N.S.), 5(4) : $152-162$

Lindley (R.P.). 1980. - Macromia gamblesi spec. nov. from the Central African Republic (Anisoptera : Corduliidae). Odonato logica, 9 (2), 189-195.

Martin (R.). 1906. - Cordulines. Collns zool. de Selys Longchamps, $17: 1-94$, pls $1-3$.

Schouteden (H.). 1934. - Catalogues raisonnés de la faune ento mologique du Congo Belge. Odonates. Annis Mus. r. Congo Belge, Zool., Ser. $3(2) 3(1): 1-84$. 